\title{
Current status and content of quantitative criteria in clinical trials of compression therapy
}

\author{
Congcong $\mathrm{LEI}^{1}$ and $\mathrm{Lu} \mathrm{CHEN}^{2}$ \\ 1,2 Institute of textile and clothing, Shanghai University of Engineering Sciences, Shanghai 201600, China
}

\begin{abstract}
Objective: The scale of the treatment effect of lymphedema pressure has not been clarified, and there appears to be a lack of evidence for research in this area. There is no clear and uniform standard for measuring the experimental results of lymphedema treatment with pressure clothing, and there seems to be a lack of research on the experimental results measure. The purpose of this study was to summarize and quality evaluate the existing scientific evaluation criteria for the therapeutic measure of upper limb compression. To review the differences and similarities in efficacy evaluation criteria of pressure cuff, pressure bandage, CDT and other treatment methods. And some new ideas. Methods: Narrative review based on search in Pub-Med/Web of Science through keywords related to compression in lymphedema. A systematic review of published literature was performed. Results: In the review, 1716 articles were screened, of which 11 trials were included. The primary end point of the assessment and results was to measure changes in arm volume, secondary endpoints were the quality of life, patient compliance, range of motion of arm joints and other reason. Although the effectiveness of compression therapy can be obtained by comparing experiments, there is no uniform measurement of experimental results, so it is impossible to compare the effectiveness between different experiments. Conclusions: At present, in the treatment of lymphedema, the effect of the pressurized sleeve is recognized, and the accepted measure is the change in arm volume, and secondary criteria vary widely. The available literature lacks a unified measurement standard for experimental results and measurement items. Researchers should combine the actual situation of the local patients with lymphedema to construct a suitable measure of the effect of lymphedema compression treatment after breast cancer.
\end{abstract}

\section{Introduction}

Lymphedema is a chronic profressive disease without a cure in women, and lymphedema of upper limb following breast cancer treatment is one of the major long-term complications[1]. According to the most recent International Society of Lymphology (ISL) consensus document[2], We can learn about the current non-operative treatment of lymphedema (Figure 1). The generally accepted schemes of the combined treatment of lymphedema of upper is physical therapy and adjuvants.

Currently, the following methods are mainly used in the literature (Intermittent pneumatic compression, Complete Decongestive Therapy (CDT), manual lymphatic drainage (MLD), Compression garments). CDT is the most effective treatment which is a combination of four methods (MLD, compression therapy, a low-stretch elastic stocking or sleeve, continued exercises and skin care) as it reduces the symptoms of lymphedema and improves patients' quality of life and psychological states [3]. Multilayer low-stretch bandages or sleeve and elastic compression is the cornerstone of CDT[4]. Although CDT and other therapeutic techniques have demonstrated positive results, but the evidence base setting a scale in the treatment effect of lymphedema pressure has not been clarified.

Scientific norms and unified experimental quantitative standards can provide more targeted treatment programs for medical staff and patients, as well as provide strong support for scientific researchers' experiments.

In this article, we introduce the entity of lymphedema treatment, as well as medical treatment with Compression therapy. We also review therapies described in the literature. The status quo and main problems of the existing clinical trials were clarified to provide reference for domestic clinical trials. 


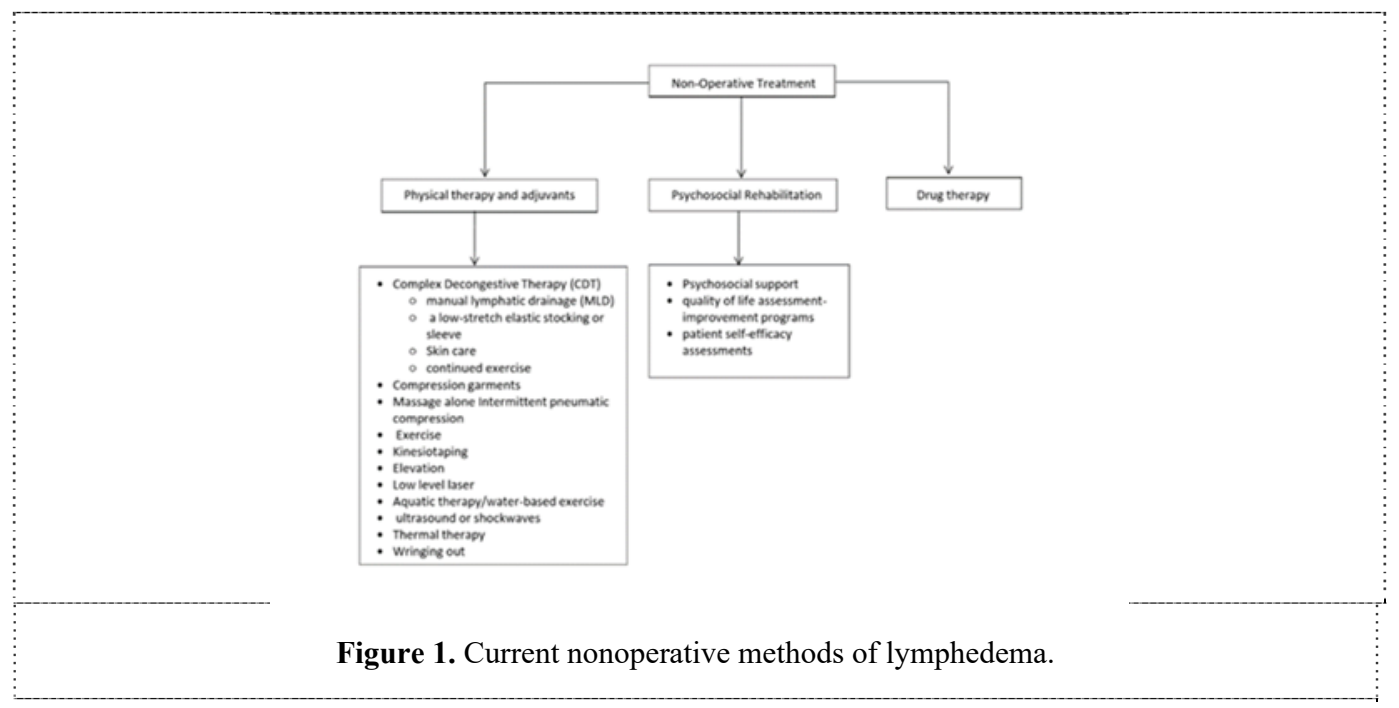

\section{Methods}

Search strategy. A systematic search of the relevant guideline and systematic review databases was undertaken between 06 May 2020 and 05 June 2020. The following databases were searched: National Center for Biotechnology Information (NCBI), National Lymphedema Network (NLN) and Web of Science. Focusing on articles of the last 5 years and key search terms included 'Upper Extremity', 'elastic hosiery', 'pressure bandages', 'compression therapy', 'compression sleeves', 'lymph', 'lymphatic disease', 'nonsurgical therapy' and 'venous disease'. Intentionally no search was performed on lower extremity and intermittent pneumatic compression, which does not apply to this review. The 2020 International Society of Lymphology (ISL) consensus document is mainly harvested from the retrieval results of NLN. Initially, the searches produced 789 results in first search strategy and 272 in second one. ( Figure 2).

Inclusion and exclusion criteria are as followed, Inclusion criteria: RCT within the last 5years, Outcome of Lymphedema, Lymphedema of upper limbs, Patients received compression sleeves or pressure bandages alone or in conjunction with MLD; exclusion criteria: without bandages or compression garments were used, without control test, preventive and therapeutic options, only discuss effectiveness and safety, discuss research method.

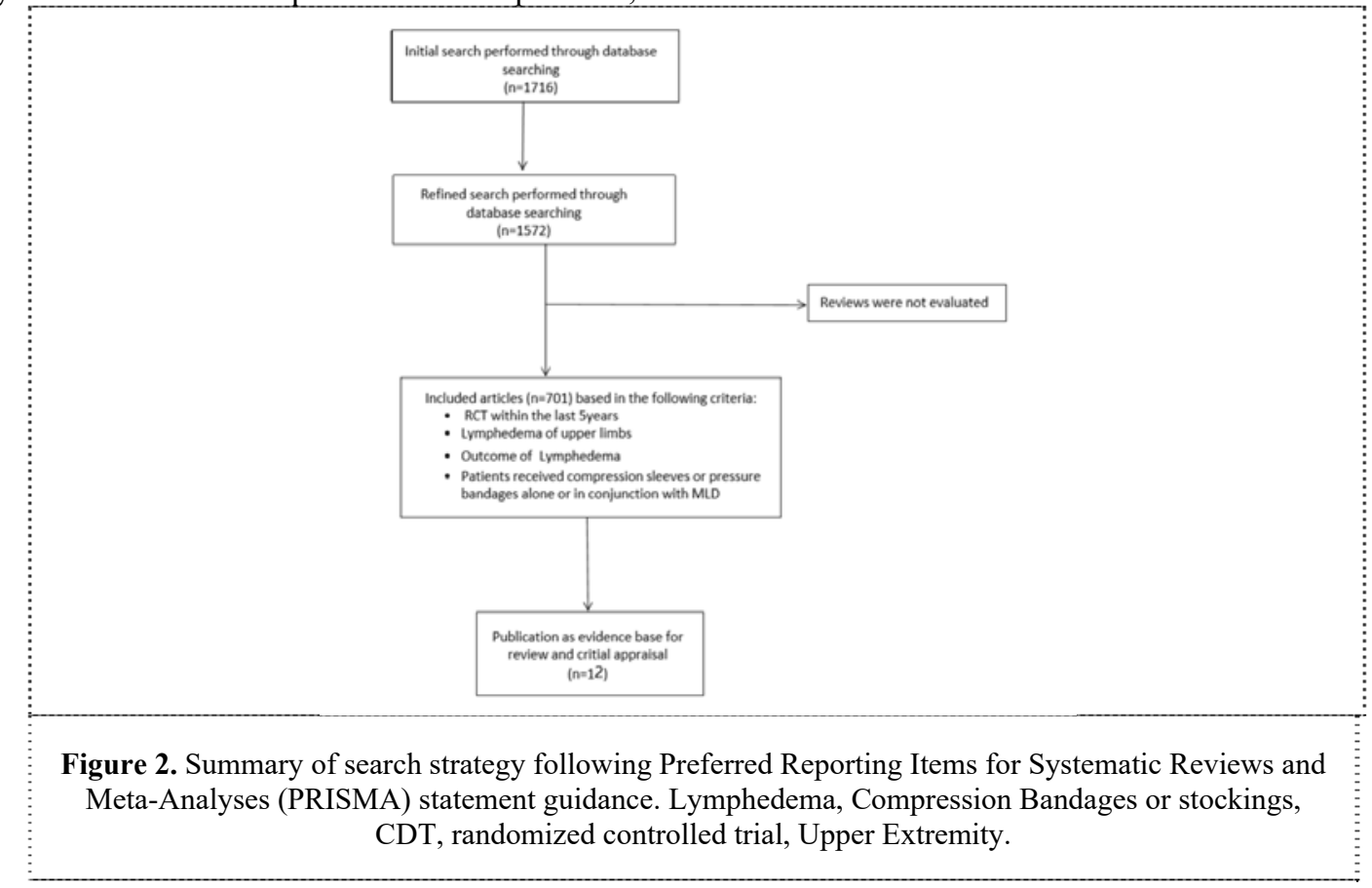

\section{Results subsection}

The results of this search strategy were further refined by analyzing the review, a total of 56 potentially relevant publications were selected. After a decision to include only literature on compression stockings and upper extremity, a further review identified 11 relevant 
publications as the evidence base for the measurement of experimental results. The characteristics of included study arms are displayed in Figure 3.

In 11 groups experiments, there are 7 groups of experimental time 3 months or less, 3 groups using CDT treatment, 5 groups used compression bandage or compressed cuff treatment. primary criterion for arm volumes or limb volume of 6 groups, and the criteria are different, Arm volume was measured by a plastic tape with light pressure $(\mathrm{V}=\mathrm{h}(\mathrm{C} 2+\mathrm{Cc}+\mathrm{c} 2) / 12 \mathrm{p})$ in two article [5] [6]. In other article limb volume using a simplified frustum formula (summed truncated cone). The edema volume 1 group, lymphedema to cure the success rate of 3 groups, and a set of the velocity of blood flow as a primary standard.
In the secondary criteria, 8 groups contain quality of life, but the evaluation criteria are different, including EQ-5D questionnaire [7], LYMQOL arm questionnaire [8], validated questionnaire [6], QLQBR23 questionnaires [9], four questions[10], visual analogue scale [11], the Lymphedema Questionnaire [5]. There are 2 group that involve compliance [9] [8], the evaluation criteria is EORTC QLQ-C30 questionnaires [9]. It also takes Ranges of motion of the shoulder (flexion, abduction, and external rotation) as the detection standard. Usually they are measured with a goniometer [5] [10].

In actual treatment, patients often choose to despise or give up treatment due to comfort and other factors, and patients' compliance in treatment should also be included in the measurement criteria [8].

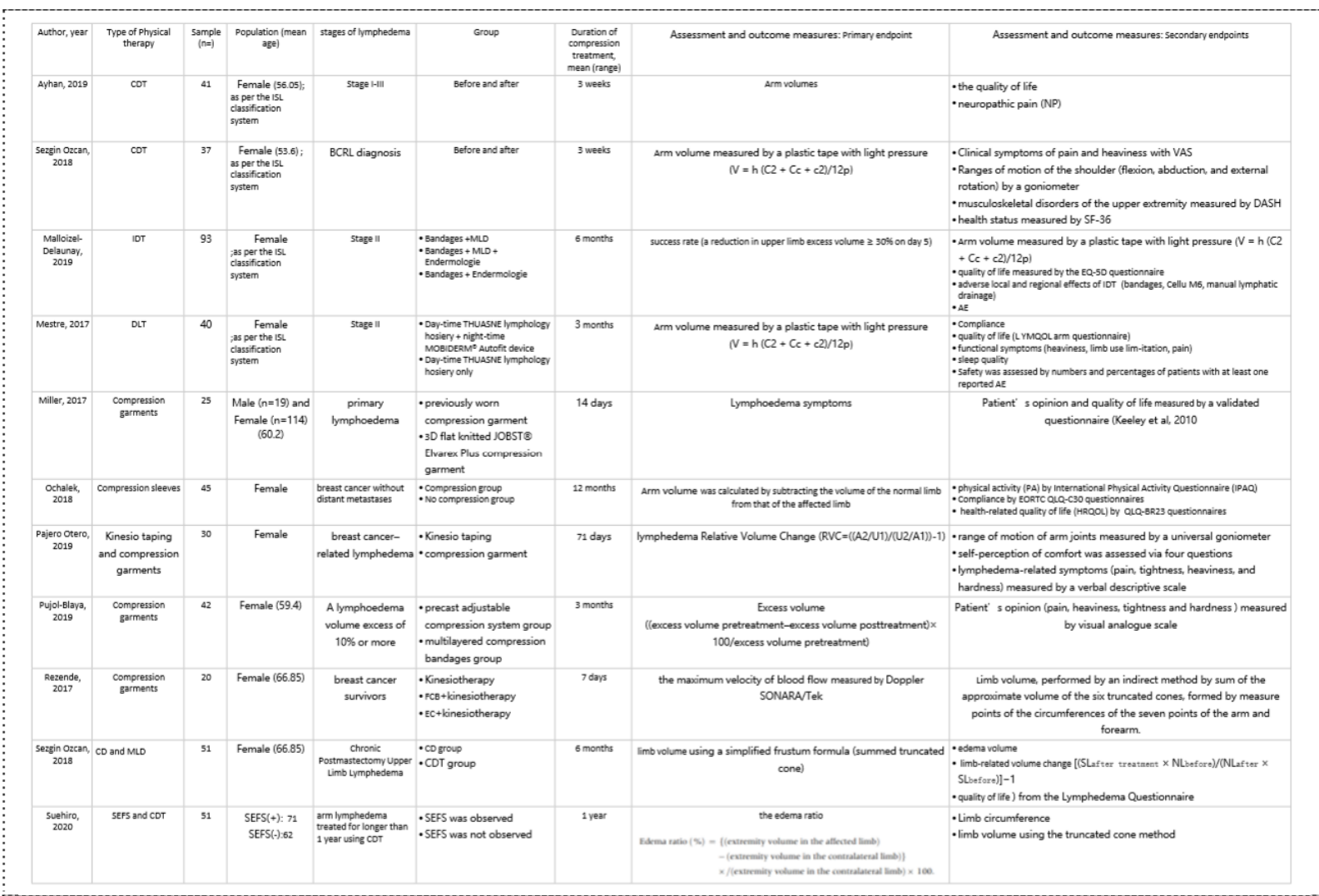

Figure 3. Characteristics of the included studies

\section{DISCUSSION}

In this review, we aimed to summarize and quality assess the existing published evidence concerning The scale of the treatment effect of lymphedema pressure. Only tests carried out in the past 5 years were included because of the rapid development of Knit Garments, as well as significant changes in treatment strategies and surgical techniques for lymphedema pressure.

There are many methods to measure the excess volume, and since the limb volume changes with the increase/decrease of the patient's weight, and the different time of measurement also has an impact, a standard measurement scale should be established. However, when upper extremity lymphedema occurs after breast cancer treatment, it may affect other parts of the body and requires targeted treatment. Therefore, to compare and contrast the effectiveness of various treatment regimens, it is best to measure them in a standardized manner.

Although pressure cuff for the treatment of lymphedema international has been confirmed, there are few domestic studies of measure scale [12], and due to the individual specificity of lymphedema, often used in the treatment of CDT effect is best [13], and the CDT treatment professionals need to follow up in 
time, and the height of the patient to cooperate, so in formulating the unified measuring scale quite difficult. The optimal pressure values for lymphedema at different stages are different. In the actual treatment, it is necessary to timely track the patient's edema status and constantly adjust the pressure suit or pressure sleeve [14].

To improve the normality and accuracy of lymphedema stress therapy, more attention should be paid to the study of treatment outcome measurement scale in future studies.

\section{References}

1 Cal and Z. Bahar, "Women's Barriers to Prevention of Lymphedema After Breast Surgery and Home Care Needs: A Qualitative Study," (in eng), Cancer Nurs, vol. 39, no. 6, pp. E17-e25, Nov/Dec 2016.

2 "The diagnosis and treatment of peripheral lymphedema: 2020 Consensus Document of the International Society of Lymphology," (in eng), Lymphology, vol. 53, no. 1, pp. 3-19, 2020.

I. Tzani, M. Tsichlaki, E. Zerva, G. Papathanasiou, and E. Dimakakos, "Physiotherapeutic rehabilitation of lymphedema: state-of-the-art," (in eng), Lymphology, vol. 51, no. 1, pp. 1-12, 2018.

3 S. Vignes, "[Lymphedema: From diagnosis to treatment]," (in fre), Rev Med Interne, vol. 38, no. 2, pp. 97-105, Feb 2017. Les lymphœdèmes : du diagnostic au traitement.

4 D. Sezgin Ozcan, M. Dalyan, S. Unsal Delialioglu, U. Duzlu, C. S. Polat, and B. F. Koseoglu, "Complex Decongestive Therapy Enhances Upper Limb Functions in Patients with Breast CancerRelated Lymphedema," (in eng), Lymphat Res Biol, vol. 16, no. 5, pp. 446-452, Oct 2018.

A. Miller, "Impact of seamless compression garments on limb functionality, comfort and quality of life," (in eng), Br J Community Nurs, vol. 22, no. Sup10, pp. S26-s37, Oct 12017.

5 J. Malloizel-Delaunay et al., "Endermology treatment for breast cancer related lymphedema (ELOCS): Protocol for a phase II randomized controlled trial," Eur J Obstet Gynecol Reprod Biol, vol. 241, pp. 35-41, Oct 2019.
6 S. Mestre et al., "Interest of an auto-adjustable nighttime compression sleeve (MOBIDERM ${ }^{\circledR}$ Autofit) in maintenance phase of upper limb lymphedema: the MARILYN pilot RCT," (in eng), Support Care Cancer, vol. 25, no. 8, pp. 2455-2462, Aug 2017.

7 K. Ochalek, T. Gradalski, Z. Szygula, and H. Partsch, "Physical Activity With and Without Arm Sleeves: Compliance and Quality of Life After Breast Cancer Surgery-A Randomized Controlled Trial," (in eng), Lymphat Res Biol, vol. 16, no. 3, pp. 294-299, Jun 2018.

8 V. Pajero Otero, E. García Delgado, C. Martín Cortijo, H. M. Romay Barrero, E. de Carlos Iriarte, and J. Avendaño-Coy, "Kinesio taping versus compression garments for treating breast cancerrelated lymphedema: a randomized, cross-over, controlled trial," (in eng), Clin Rehabil, vol. 33, no. 12, pp. 1887-1897, Dec 2019.

9 V. Pujol-Blaya, S. Salinas-Huertas, M. L. Catasús, T. Pascual, and R. Belmonte, "Effectiveness of a precast adjustable compression system compared to multilayered compression bandages in the treatment of breast cancer-related lymphoedema: a randomized, single-blind clinical trial," (in eng), Clin Rehabil, vol. 33, no. 4, pp. 631-641, Apr 2019.

10 R. Hansdorfer-Korzon, J. Teodorczyk, A. Gruszecka, J. Wydra, and P. Lass, "Relevance of low-pressure compression corsets in physiotherapeutic treatment of patients after mastectomy and lymphadenectomy," (in eng), Patient Prefer Adherence, vol. 10, pp. 1177-87, 2016.

11 T. I. Grushina, "[What physiotherapeutic method for the treatment of post-mastectomy lymphedema is the most effective?]," (in rus), Vopr Kurortol Fizioter Lech Fiz Kult, vol. 94, no. 4, pp. 59-66, 2017. Kakoǐ metod fizioterapii postmastéktomicheskor limfedemy naibolee éffektiven?

12 M. Karafa, A. Karafova, and A. Szuba, "The effect of different compression pressure in therapy of secondary upper extremity lymphedema in women after breast cancer surgery," (in eng), Lymphology, vol. 51, no. 1, pp. 28-37, 2018. 